\title{
Développement durable et territoires
}

\section{Mobilisations écologiques actuelles, mobilisations des années 1960-1970 : quels parallèles ?}

Current ecological mobilizations and mobilizations of the 1960-1970's: are there some parallels?

\section{Edwin Zaccai et Alexandre Orban}

\section{OpenEdition Journals}

Édition électronique

URL : http://journals.openedition.org/developpementdurable/11847

DOI : 10.4000/developpementdurable. 11847

ISSN : 1772-9971

Éditeur

Association DD\&T

\section{Référence électronique}

Edwin Zaccai et Alexandre Orban, « Mobilisations écologiques actuelles, mobilisations des années 1960-1970 : quels parallèles ?», Développement durable et territoires [En ligne], Vol. 8, n² I Juillet 2017, mis en ligne le 28 juillet 2017, consulté le 20 avril 2019. URL : http://journals.openedition.org/ developpementdurable/11847; DOI : 10.4000/developpementdurable.11847

Ce document a été généré automatiquement le 20 avril 2019

\section{cc) (†) (8)}

Développement Durable et Territoires est mis à disposition selon les termes de la licence Creative Commons Attribution - Pas d'Utilisation Commerciale 4.0 International. 


\title{
Mobilisations écologiques actuelles, mobilisations des années 1960-1970 : quels parallèles?
}

\author{
Current ecological mobilizations and mobilizations of the 1960-1970's: are there
} some parallels?

Edwin Zaccai et Alexandre Orban

1 Même s'il a existé des formes multiples de mobilisations environnementales dans l'histoire des sociétés humaines, il convient généralement de faire remonter les mouvements écologistes au sens occidental moderne aux années 1960-1970, aux ÉtatsUnis et en Europe. Ces courants et les idées qui y sont associées ont modifié les sociétés de façons diverses, ainsi que leurs impacts sur l'environnement. Des formes d'institutionnalisation importantes se sont produites, sous l'égide du développement durable. Pourtant une mouvance d'ordre plus radical, expérimental et moins institutionnalisé se manifeste également au long des décennies.

2 Cet article jette un regard rétrospectif sur des mobilisations écologiques des années 1960-1970 et les compare avec certains aspects de la situation actuelle en France et aux États-Unis. Il met à jour des points communs, mais aussi des différences : ce « point de vue » n'a d'autre ambition que de tracer quelques repères pour la réflexion à travers une mise en perspective, nécessairement partielle, à quarante ans de distance.

Pour ce faire, nous traitons de la différence des contextes par rapport aux questions environnementales, des thèmes dominants dans le débat, de quelques auteurs inspirant ces mobilisations, d'aspects sociodémographiques, du type d'engagement dans des alternatives et enfin des rapports avec la politique organisée. Nos sources sont essentiellement des ouvrages parus aux différentes époques et des auteurs qui analysent eux-mêmes ces mouvements. 


\section{Préoccupations des populations par rapport aux problèmes d'environnement}

Contrairement à une idée répandue aujourd'hui, les préoccupations environnementales ne constituent pas une caractéristique plus importante à notre époque que voici quelques décennies. Les cas des États-Unis et de pays européens de l'ouest des années 1960-1970 se distinguent en ce sens par l'expression massive de revendications environnementales largement répandues. En témoignent les manifestations pour le Earth Day qui ont réuni des millions de personnes aux États-Unis ou les sondages Eurobaromètre plaçant l'environnement comme première priorité des sondés en 1973 (CCE 1983).

5 Si cette période et notre réalité contemporaine mettent en avant des questions environnementales comme préoccupantes, il est cependant important de différencier celles-ci. En effet, les années 1960-1970 s'accompagnent d'une visibilité accrue des problèmes environnementaux par une série d'impacts directs sur les sociétés humaines : pollutions de l'air urbain, déchets dans l'environnement immédiat, pollutions visibles des fleuves, etc. (Zaccai, 2011). Aujourd'hui, de grands impacts environnementaux sont, a contrario, relativement éloignés des réalités occidentales quotidiennes, tels les changements climatiques ou la perte sévère de biodiversité tropicale, ce qui influe sur la perception de ces problèmes et les types de mobilisations. Il y aurait d'ailleurs à cet égard des parallèles à trouver entre les mobilisations américaines contre les pollutions urbaines des années 1960-1970 et la situation contemporaine de la Chine, où les protestations sont nombreuses contre des pollutions directement nuisibles, de l'air en particulier (sans commune mesure avec la situation actuelle en France).

\section{Perspectives d'effondrements}

6 Une autre différence marquante entre les périodes est la représentation de menaces d'« effondrement» de la société pour des raisons écologiques au sens large (rareté, pollutions). Cette perspective a été propagée de façon éminente après 1972 par le rapport de Club de Rome Halte à la croissance (The Limits to Growth, Meadows et al., 1972), l'un des livres les plus influents dans l'histoire des idées écologiques. Avec des projections de scénarios d'effondrement en cas de poursuite de la croissance mondiale, ces thèses tranchaient avec les tendances sans précédent de croissance du niveau de vie moyen qui avaient prévalu en Occident depuis la Seconde Guerre mondiale. L'ouvrage a été discuté à l'époque de sa parution et a influencé une série d'auteurs, toutefois avant la crise du pétrole de 1973 il semblait bien établi dans l'opinion que la génération montante aurait de meilleures conditions de vie que celle en place.

7 Cette croyance s'est à présent massivement inversée ${ }^{1}$. Actuellement, les chercheurs intéressés par l'environnement qui ne pronostiquent pas de dégradations importantes au $\mathrm{XXI}^{\mathrm{e}}$ siècle sont devenus rares. Les projections sont alimentées par de très abondants travaux scientifiques, tant sur le climat que sur la biodiversité et dans une moindre mesure, l'exploitation des ressources naturelles - à l'inverse des priorités du rapport au Club de Rome. À cela s'ajoutent d'autres fragilités, notamment financières et technologiques, sur lesquelles là aussi les travaux se multiplient comme on le voit dans des recherches qui en font des recensions (Semal, 2012 ; Stevens, Servigne, 2015). Enfin ce 
parfum de « déclin » est alimenté en Europe et aux États-Unis par une perte relative de pouvoir économique de ces régions face aux puissances émergentes telles que la chine ou l'Inde, voire par la communication abusive autour des menaces d'immigration. Les récentes évolutions vers des votes populistes dans des pays occidentaux illustrent entre autres des peurs de "décroissances » supplémentaires de niveau de vie pour certaines parties de la population. Ce sont des éléments de toile de fond que nous retrouverons en parlant des mobilisations actuelles.

\section{Auteurs emblématiques}

8 Un des facteurs d'influence des mouvements sociaux réside dans une production culturelle originale à l'époque des débuts de l'écologie politique. Des auteurs influents tels qu'Ivan Illich, André Gorz ou René Dumont ont ainsi contribué à orienter la pensée environnementale et ses mobilisations. Ces trois auteurs ont certes des différences. Illich $(1977)^{2}$ vient du monde de la théologie, il pourfend le « développement » par rapport aux modes de vie traditionnels (sans doute influençant l'image d'une écologie de « retour à la bougie » pour longtemps en France). Gorz (1978) s'inspire du marxisme, pense le partage du travail et des gains de productivité. Dumont (1973), qui contribue fortement à façonner l'écologie politique en France durant ces années (Vrignon 2017) s'intéresse à l'agriculture et au Tiers-Monde à une époque où les mobilisations sont surtout locales et nationales. Mais ils s'inspirent l'un l'autre, ainsi que beaucoup d'autres, et ont en commun de prôner une refonte importante de la société dans son ensemble, loin de se limiter à des réformes environnementales. Pour s'en convaincre on peut consulter par exemple le livre Écologie: détente ou cycle infernal (1973) de Pierre Samuel, président des Amis de la Terre en France à cette époque, qui témoigne de l'essai d'articulation de l'écologie avec d'autres causes, féminisme, pacifisme, médecines « douces », etc.

L'environnementalisme gestionnaire et la « modernisation écologique » qui vont dominer les années 1990 et 2000 se situent bien loin de ces pères fondateurs. Prônant l'efficience des ressources et se gardant de se prononcer sur les valeurs et même sur les conflits politiques, ils vont nourrir la branche la plus consensuelle du développement durable, facilement récupérable par le monde de l'entreprise et de la finance. C'est contre cette tendance que des auteurs français comme Serge Latouche (2007) - qui se revendique explicitement de Illich - ou Pierre Rabhi (2010) vont prôner le thème de la décroissance, sous différentes formes, suivis de Rob Hopkins avec le thème de la transition (Hopkins, 2014). Ces auteurs, chacun avec leurs accents particuliers, cherchent à refonder la société en privilégiant le niveau local (avec un accent important mis sur l'agriculture) et les réseaux humains directs par rapport aux institutions. Dans le contexte contemporain, où les menaces d'effondrement sont plus répandues, ces idées vont recueillir beaucoup de succès et nourrir des mobilisations écologiques. Notons aussi la mise en évidence plus affirmée de valeurs d'authenticité que dans la période précédente, ce qui peut faire penser aux débuts de l'écologie politique.

\section{4. Éléments sociodémographiques}

10 La «contre-culture » et les mouvements massifs de la jeunesse aux États-Unis et en France (entre autres) à la fin des années 1960, sont associés au baby-boom, à la hausse généralisée du niveau de vie et à une croissance sans précédent de l'inscription à 
l'université. Selon des auteurs qui se réfèrent aux courants du « post-matérialisme » et de la théorie des Nouveaux Mouvements Sociaux, dont Ronald Inglehart et Alain Touraine sont respectivement des figures de proue, la satisfaction de besoins matériels primaires liée aux politiques de consommation de masse, le temps disponible de la jeunesse notamment, et le développement de valeurs post-matérielles au travers de l'éducation ont alimenté la contre-culture et l'écologie radicale.

11 Si ces facteurs ont certainement joué, il faut cependant nuancer cette grille explicative. Les caractères «nouveau » et "qualitatif » attribués à la contre-culture des années 1960-1970 sont par exemple remis régulièrement en cause (Neveu, 2011). D’autres travaux déclinent dès cette époque différents types de mobilisations environnementales selon les catégories sociales engagées. Ainsi Gould et al. (2004) réalisent un historique de relations entre intérêts de catégorie sociales et mobilisations environnementales aux États-Unis, tenant compte du fait que la situation hiérarchique dans la société influence les conditions matérielles, mais également les représentations et valeurs. Pour la France, Vrignon (2017) s'attache à documenter divers courants en interaction (y compris conservateurs) à la base de l'écologie politique. Quant à l'analyse très riche et documentée à laquelle se livre Cefaï (2007), tout en offrant également d'autres prismes explicatifs que la théorie des nouveaux mouvements sociaux, elle fournit des éléments troublants à celui qui cherche des similitudes entre la période des années 1960-1970 et l'époque actuelle, à travers « la réinvention des styles de vie » (p. 449), « l'utopie concrète au quotidien » (ibid.), «l'invention du présent» (p. 426), ceci aussi parce que selon l'auteur «les utopistes étaient nombreux à expérimenter de nouveaux espaces de vie » (p. 433).

12 À notre époque, il subsiste une croissance de l'augmentation des inscrits à l'université, mais c'est surtout le chômage des jeunes, y compris de diplômés et la dégradation relative ou absolue - du niveau de vie des classes moyennes qui peuvent être soulignés. Ceci notamment dans le sillage de la crise économique de 2009 et, pour l'Europe, davantage dans les pays méditerranéens. Cette situation, sur un arrière-fond de dégradations présentes et à venir, écologiques et économiques, incite à des mobilisations qui soient à la fois basées sur des valeurs et sur une recherche pratique de modes de vie si pas durables, au moins « résilients », et c'est ce que nous allons voir au point suivant.

\section{Alternatives concrètes}

Dans les années 1960-1970, il y a eu des expériences concrètes plus ou moins éphémères de « retour à la terre » dont témoignent aujourd'hui des photos jaunies d'ancêtres à longs cheveux, et notamment aux États-Unis où l'imagerie des pionniers est plus vivace qu'en France. $\mathrm{Y}$ a-t-il des parallèles et des différences à trouver avec l'imaginaire et les pratiques de mouvements d'alternatives concrètes actuelles?

14 Tout d'abord, la mise en réseau est évidemment particulièrement forte aujourd'hui grâce à Internet et aux réseaux sociaux électroniques. Par ailleurs, une internationalisation des luttes s'est imposée comme une donnée bien plus courante que dans les années 1960-1970, où seule une infime minorité de jeunes Européens ou d'Américains avait voyagé hors de leur continent.

15 Ensuite, une perspective que nous qualifierons de doublement «survivaliste " s'est davantage ancrée dans les alternatives concrètes actuelles. Doublement, car il s'agit d'une 
part d'une volonté de résilience face à des déclins écologiques ou autres, et d'autre part d'une recherche de moyens matériels de subsistance (créer son activité). En guise d'exemple, l'initiative de Villes en transitions anticipait à l'origine la rareté du pétrole. Malgré le fait que la question climatique prenne une place dominante aujourd'hui (il y a actuellement trop de réserves d'énergies fossiles par rapport aux plafonds d'émissions prescrits par les scientifiques pour limiter le réchauffement global à deux degrés) le succès de ce mouvement ne s'est pas démenti, bien au contraire. En effet, l'ambition est bien de se raccorder au local, et tout particulièrement à l'agriculture, d'y "faire société » dans le sens d'une recherche évidente de convivialité, mais aussi d'y trouver des moyens concrets de vie (Semal, 2012). Le chômage des jeunes, le peu de perspectives que beaucoup voient dans la poursuite des tendances économiques, le développement des connaissances et techniques écologiques (en énergie renouvelable, en agriculture, etc.) et une certaine demande en la matière (bio, circuits courts, etc.) s'allient pour stimuler la recherche d'alternatives viables, qui sont bien illustrées par le film à succès Demain. Certains sociologues évoquent un "alteractivisme " spécifique à une génération jeune, passant par un engagement fort et concret en accord avec leurs valeurs, et ce, dans de multiples pays (Pleyers et Capitaine, 2016).

\section{Rapport à la politique}

Comme nous l'avons vu, les idées de certains précurseurs de l'écologie radicale dépassaient de loin l'écologie gestionnaire. Leur ambition était politique au sens large. Nous retrouvons ce type de réflexions dans les mouvements écologiques des années 1960-1970, au travers des critiques de la croissance, du système démocratique, des solutions technologiques, des grandes entreprises ou encore du capitalisme. La légitimité de l'État en tant que garant de l'intérêt public et du progrès sociétal, qu'il a pu incarner au tournant de la Seconde Guerre Mondiale dans les pays occidentaux, est alors remise en cause. À ce titre, les mouvements écologistes de l'époque porteront haut des revendications d'autonomie, de participation à la décision et de contre-expertise (Lechat, 2014).

17 Depuis, nombre de mouvements écologistes sont entrés en politique (Boy et al. 1995) où ils ont contribué en effet à promouvoir ce type de valeurs. Ces principes ont bénéficié de la hausse du niveau d'instruction dans la population, tout en étant transformés par les moyens informatiques de communication. La portée des contre-expertises est significativement modifiée à une époque où l'accès aux documents est sans commune mesure par rapport au passé et où d'innombrables commentaires sont partagés constamment sur tous les sujets. La participation institutionnalisée a aussi montré des faiblesses démocratiques, limitant significativement l'implication des personnes dans la vie politique. En outre, la participation populaire et locale n'entraîne pas nécessairement la revendication de valeurs progressistes comme semblaient s'y attendre les écologistes des débuts. L'utilisation de réseaux sociaux et de campagnes participatives alimentant des objectifs conservateurs ou de rejet de certaines parts de la population en offre des exemples ces dernières années.

18 À présent, la participation semble se porter de plus en plus sur la mise en place d'initiatives alternatives à celles de l'État, quitte à espérer des mouvements de contagion et d'institutionnalisation (théorie de la transition), sans toujours théoriser justement les voies sociopolitiques et économiques de cette transition. La perception d'un 
affaiblissement de l'action positive de l'État est en partie liée au contexte d'érosions de toutes sortes déjà pointé. Pour Pech (2017), il existe en France aujourd'hui un climat d'« insoumission» qui dépasse largement la critique écologiste, avec notamment des votes « anti-systèmes » de tout bord. Une partie de ces discours a singulièrement à voir aussi avec la critique du néolibéralisme et du pouvoir des grandes entreprises. En effet, l'expansion historique de leur influence constitue l'un des faits les plus marquants qui a remodelé les sociétés occidentales. Dès lors, le capitalisme est devenu un objet beaucoup plus net de critique qu'avant la dernière crise économique, ce qui n'est d'ailleurs pas sans rappeler des réflexions de Mai 1968 en France (Baudrillard 1970) ou de la contre-culture aux États-Unis. Cette tendance s'observe aujourd'hui dans différentes luttes en France sous l'égide de l'acronyme ZAD (Zones À Défendre), telles que les exemples de l'aéroport de Notre-Dame-Des-Landes ou le barrage de Sivens. Des observateurs y relèvent un réel durcissement des conflits environnementaux (Subra, 2015). C'est aussi le cas aux ÉtatsUnis, comme cela est bien documenté dans le livre à succès de Naomi Klein sur le climat (2014), véritable manuel de stratégie pour des mobilisations écologistes contemporaines. Le contexte post-élection du président Trump risque sans doute de renforcer cette effervescence d'activisme en partie confrontationnel.

\section{Conclusion}

La présente rétrospective a permis de retracer plusieurs distinctions et similitudes entre des mobilisations écologiques contemporaines et celles des années 1960-1970. Quatre aspects majeurs de contexte diffèrent: (i) les types de problèmes environnementaux essentiels, (ii) le contexte socio-économique (iii) la perspective d'«effondrements» multidimensionnels et (iv) l'institutionnalisation de certains principes et actions environnementaux. Mais des similitudes existent sur les plans de l'engagement personnel, des expérimentations avec une visée de transformation sociale, ou encore de l'intérêt pour le local. L'État reste l'objet de critiques, mais aussi les entreprises capitalistes. Les formes de mobilisations font apparaître à la fois désobéissance civile et modes de vie alternatifs.

Au-delà d'une curiosité scientifique et d'un rappel de données du passé, la comparaison historique entre ces deux périodes nous invite à nous questionner sur les mobilisations écologiques contemporaines, et leur double mouvement de radicalisation et d'institutionnalisation (Ollitrault, Villalba, 2014). De nombreuses voix se sont interrogées sur le fait de savoir comment ces revendications pourraient gagner en puissance pour aboutir à davantage de résultats dans le contexte écologique inquiétant rappelé dans cet article. Sans pouvoir répondre à cette question qui dépasse largement le cadre de cet article, pointons pour terminer une voie présente dans les mouvements militants, celle d'une « convergence des luttes ». L'approche historique utilisée par Kenneth et al. (2004) à propos de coalitions entre mouvements du travail et écologiques dénote un intérêt certain en ce sens. Ce type d'analyse, intégrant de manière interdisciplinaire des rapports de catégories sociales à des éléments historiques et géographiques, mériterait une plus grande attention dans le traitement des mobilisations liées à l'écologie au sens large. 


\section{BIBLIOGRAPHIE}

Baudrillard J., 1970, La Société de consommation, Paris, Denöel.

Boy, D., Jacques V., Roche A., 1995, L'Écologie au pouvoir, Paris, Presses de Sciences Po.

Cefaï, D., 2007, Pourquoi se mobilise-t-on ?, Paris, La Découverte.

Commission des Communautés Européennes, 1983, Les Européens et leur environnement, Bruxelles.

Dumont R., 1973, L'Utopie ou la mort, Paris, Seuil.

Gorz A., 1978, Écologie et politique, Paris, Seuil.

Gould, K. A., Lewis, T. L., Roberts, J. T., 2004, "Blue-Green Coalitions : Constraints and Possibilities in the Post 9-11 Political Environment", Journal of World-Systems Research, vol. 10, ㄲo1, p. 91-116.

Hopkins R., 2014, Ils changent le monde! 1001 initiatives de transition écologique, Paris, Seuil.

Illich I., 1977, Le Chômage créateur, Paris, Seuil.

IPSOS/CGI, 2013, Les Européens et la sortie de crise, http://www.ipsos.fr/decrypter-

societe/2013-05-07-europeens-et-sortie-crise.

Klein, N., 2015, Tout peut changer. Capitalisme et Changement Climatique, Arles, Actes Sud.

Latouche S., 2007, Petit traité de la décroissance sereine, Paris, Mille et une nuits.

Lechat B., 2014, Écolo. La démocratie comme projet, Namur, Etopia.

Meadows DH., Meadows DL., Randers J. Behrens W., 1972, Limits to Growth, New York, Universe Books.

Neveu É., 2015 (6 è ed.), Sociologie des mouvements sociaux, Paris, La Découverte.

Ollitrault S., Villalba B., 2014, « Sous les pavés, la Terre. Mobilisations environnementales en France (1960-2011), entre contestation et expertise » in Pigenet M., Tartakowsky D. (dir.), Histoire des mouvements sociaux en France. De 1814 à nos jours, Paris, La Découverte, p. 716-723.

Rabhi P., 2010, Vers la sobriété heureuse, Arles, Actes Sud.

Pech T., 2017, Insoumissions. Portrait de la France qui vient, Paris, Seuil.

Pleyers G., Capitaine B., 2016, « Introduction. Alteractivisme : comprendre l'engagement des jeunes », Agora débats/jeunesses 2016/2 (N 73), p. 49-59, DOI 10.3917/agora.073.0049.

Samuel P., 1973, Écologie : détente ou cycle infernal, Paris, 10/18.

Semal L., 2012, Militer à l'ombre des catastrophes. Contribution à une théorie politique environnementale au prisme des mobilisations de la décroissance et de la transition, Thèse de doctorat, Science Politique, Lille 2, Lille.

Servigne P., Stevens R., 2015, Comment tout peut s'effondrer. Petit manuel de collapsologie à l'usage des générations présentes, Paris, Seuil.

Subra Ph., 2015, « Le durcissement des conflits environnementaux », Le Monde, 7/06/2015.

Vrignon A., 2017, La Naissance de l'écologie politique en France. Une nébuleuse au cour des années 68, Presse Universitaire de Rennes. 
Zaccai E., 2011, 25 ans de développement durable, et après ?, Paris, Presses Universitaires de France.

\section{NOTES}

1. En France $72 \%$ estiment que « Lorsque vos enfants auront votre âge » ils vivront « moins bien que vous à votre âge » (IPSOS 2013). Pour l'Europe le taux est de $51 \%$.

2. Pour chacun des auteurs de cette section 3 nous signalons un ouvrage, pour rester succincts, mais d'autres de leurs ouvrages sont pertinents également pour notre propos.

\section{RÉSUMÉS}

Cet article tente de jeter un regard rétrospectif sur des mobilisations écologiques des années 1960-1970, à la base de l'écologie occidentale moderne, et dresse des comparaisons avec certains aspects de la situation actuelle en France et aux États-Unis. Pour ce faire l'article traite, pour chacune des époques, des contextes par rapport aux questions environnementales, des thèmes dominants dans le débat, de quelques auteurs inspirant ces mobilisations, d'aspects sociodémographiques, du type d'engagement dans des alternatives et enfin des rapports avec la politique organisée. Bien que les contextes soient différents, nous relevons des similitudes sur le plan de l'engagement personnel, d'expérimentations avec une visée de transformation sociale d'ensemble, ou encore de l'intérêt pour le local.

This paper presents a short overview on 1960-1970s ecological mobilizations in France and the United States, at the origins of western contemporary ecology, and compares them with current mobilizations along several features. For both periods we analyze the main environmental issues at stake, the dominant subjects that are debated, some key authors inspiring those mobilizations, the main sociodemographic trends and the relations between the mobilizations and the political system. Despite different contexts we can highlight similarities on local-based actions, personal commitments, and experiments aiming at a radical transformation of the society.

\section{INDEX}

Mots-clés : transition, développement durable, mobilisation, associations, écologie, politique, réseaux

Keywords : transition, sustainable development, mobilization, associations, ecology, politics, networks

\section{AUTEURS}

\section{EDWIN ZACCAI}

Edwin Zaccai est professeur à l'Université Libre de Bruxelles. Il dirige le Centre d'Etudes du Développement Durable. Il a réalisé de nombreuses publications sur les relations entre 
environnement et société. Voir son site sur http://homepages.ulb.ac.be/ ezaccai/.

ezaccai@ulb.ac.be

\section{ALEXANDRE ORBAN}

Alexandre Orban est diplômé du Master en environnement de l'Université Libre de Bruxelles (ULB). Il s'intéresse aux mouvements sociaux dans des contextes urbains. Il a participé à la COP22 dans le cadre du projet Inside COP (http://www.ulbinsidecops.com/). alxorban@gmail.com 